To the Editors:

\title{
Gas under the diaphragm in xray film may mislead
}

Pneumoperitonium is suspected when radiographs show the presence of gas under the diaphragm. Recognised causes of pneumoperitoneum include perforation of a viscus, recent abdominal surgery, peritoneal dialysis and Fallopian tube insufflation. Interposition of bowel, usually hepatic flexure of the colon, between the upper border of liver and the right dome of diaphragm, known as Chilaiditis syndrome, may mimic gas under the diaphragm. It occurs in a small number of people with chronic lung diseases, cirrhosis of the liver and ascites $(1,2)$.

A previously healthy, 82-year-old man was admitted to the Teaching Hospital Karapitiya with intermittent abdominal pain, vomiting, constipation and low grade fever of two weeks' duration. He had consumed alcohol (about 5 units daily) for a period of 20 years.

On admission he was ill looking and dehydrated, but pulse rate and blood pressure were normal. The abdomen was uniformly distended with diffuse tenderness and guarding. Bowel sounds were faint. The rectum was empty and no free fluid was found in the abdomen. Initial investigations showed neutrophil leucocytosis and reduced total serum protein and albumin. Renal functions were normal. Chest radiographs revealed gas under the right diaphragm (Figure) with normal heart and lung shadows. No fluid levels were seen in an erect xray of the abdomen.

A provisional diagnosis of acute abdomen with perforation of bowel was made and the patient underwent exploratory laparotomy.

During the operation, no perforation of a viscus was seen. His pancreas was inflamed and edematous suggesting acute pancreatitis. An oedematous loop of large bowel was found between the liver and the right dome of diaphragm, and this explained the appearance of gas under the right diaphragm seen in the chest xray.

The diagnosis of pneumoperitoneum is strong when gas is seen under the both domes of diaphragm. If gas is seen only on the right side, the possibility of Chilaiditis syndrome should be considered. This condition can be safely ignored when detected as an accidental finding in a chest radiograph of a patient with unrelated illness. But it can mislead when a patient has acute abdominal pain.

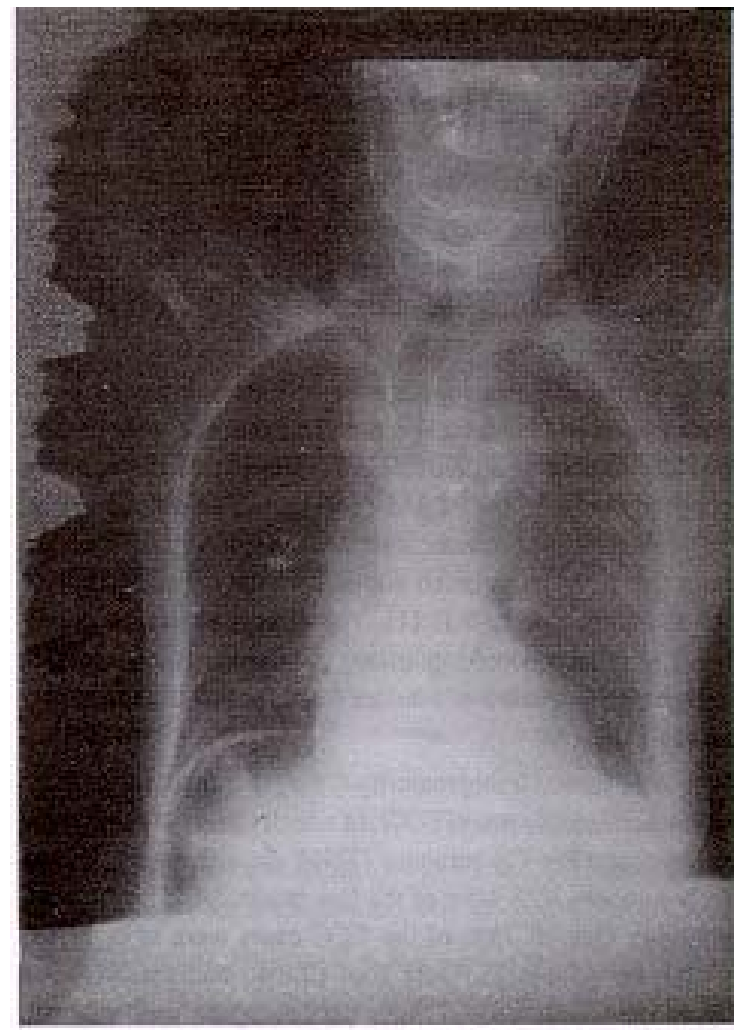

Figure. Gas under the right hemidiaphragm in an erect chest radiograph.

\section{References}

1. Grainger RG, Allison DJ: eds. Diagnostic radiology. An AngloAmerican textbook of imaging. Churchill Livingstone, London. 1986: p733.

2. Sutton David: ed. Textbook of radiology and medical imaging 5th Ed. Churchill Livingstone, London. 1993: p885.

S Lecamwasam, J D V C Lecamwasam and S Liyanarachchi, Teaching Hospital, Karapitiya, Galle, Sri Lanka. 